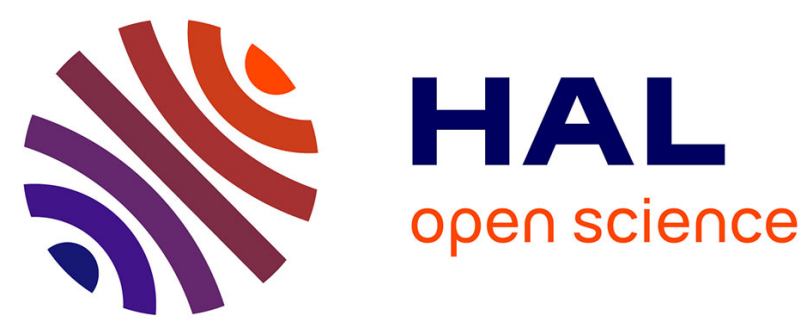

\title{
INTERACTION OF GEOMETRICAL KINKS WITH HYDROGEN FOR SCREW DISLOCATIONS IN IRON
}

\author{
J. San Juan, M. Nó, Gilbert Fantozzi, C. Esnouf
}

\section{To cite this version:}

J. San Juan, M. Nó, Gilbert Fantozzi, C. Esnouf. INTERACTION OF GEOMETRICAL KINKS WITH HYDROGEN FOR SCREW DISLOCATIONS IN IRON. Journal de Physique Colloques, 1987, 48 (C8), pp.C8-191-C8-196. 10.1051/jphyscol:1987826 . jpa-00227130

\section{HAL Id: jpa-00227130 https://hal.science/jpa-00227130}

Submitted on 1 Jan 1987

HAL is a multi-disciplinary open access archive for the deposit and dissemination of scientific research documents, whether they are published or not. The documents may come from teaching and research institutions in France or abroad, or from public or private research centers.
L'archive ouverte pluridisciplinaire HAL, est destinée au dépôt et à la diffusion de documents scientifiques de niveau recherche, publiés ou non, émanant des établissements d'enseignement et de recherche français ou étrangers, des laboratoires publics ou privés. 


\title{
INTERACTION OF GEOMETRICAL KINKS WITH HYDROGEN FOR SCREW DISLOCATIONS Iro IRON
}

\author{
J. SAN JUAN, M.L. Nó, G. FANTOZZI* and C. ESNOUF* \\ Dpto de Física del Estado Sólido, Facultad de Ciencias, \\ Universidad del Pais Vasco, Aptdo 644, SP-48009 Bilbao, Spain \\ * Groupe d'Etudes de Métallurgie Physique et de Physique des \\ Matériaux, LA-341, INSA Bât. 502, F-69621 Villeurbanne Cedex, \\ France
}

\begin{abstract}
Resumé - Les mesures de frottement intérieur et de module realisées à basse température pour différentes concentrations en hydrogène ont montré que la composante $\alpha$ "du pic $\alpha$ dans le fer apparait, pour un large domaine de concentrations en hydrogène, au même temps que la relaxation Snoek-Koster (H). Ces résultats nous permettent de conclure que la mobilité des décrochements géométriques sur les dislocations vis est très peu modifiée par les atomes d hydrogène. Néanmoins, l'interaction des décrochements gémétriques sur les dislocations vis avec des amas d hydrogène diatomiques est importanteet donne lieu à un processus de relaxation qui participe à la composante $S K 2$ de la relaxation $S K(H)$.
\end{abstract}

Abstract - Measurements of internal friction and modulus at low temperature for different amounts of hydrogen have shown that the $\alpha$ ' component of the $\alpha$ peak in Iron can appear simultaneously with the $S-K(H)$ peak for a large range of amount of hydrogen. These results allow us to conclude that the geometrical kink mobility on screw dislocations is only weakly modified by the hydrogen atoms. Nevertheless the interaction of geometrical kinks on screw dislocations and diatomic hydrogen clusters is noticeable and causes a relaxation process that contributes to the SK2 component of $S K(H)$ relaxation.

\section{I.- INTRODUCTION}

The intrinsic mobility of dislocations is strongly modified by means of the presence of hydrogen in iron. Indeed, the $\alpha$ peak, due to kink pair formation (KPF) on non-screw dislocations, disappears to give way to Snoek-Köster(H) relaxation which is constituted by two components, SK1 around $110 \mathrm{~K}$ and SK2 at about $155 \mathrm{~K}$ (for $1 \mathrm{~Hz}$ ). The experimental characteristics of the SK(H) relaxation have been studied in various papers (1-9). The theoretical intexpretation of these components has been reviewed in a recent paper (10), which gives a new analysis of the SK(H) relaxation according to all experimental characteristics. In this sense, it can be stated that the SK1 component is due to thermally activated kink formation on the non-screw dislocations controlled by the diffusion of the hydrogen atoms situated along the dislocation line. A more precise analysis of SK2 component, which historically has aroused more controversy, has allowed us to exclude the interpretations of KPF on the screw dislocations controlled by the diffusion of the hydrogen atoms, which were proposed by Hirth (11), as well as the longitudinal diffusion of the hydrogen atoms proposed by Ritchie (12). In this way, we have pointed out that the SK2 component is due to the same process as the SK1, but is controlled by the diffusion of small clusters of hydrogen (probably diatomic in nature). On the other hand, recently, internal friction experiments with a static stress have clearly shown that the $\alpha^{-}$component situated on the low temperature side of the $\alpha$ peak can be attributed to the geometrical kink migration (GKM) on screw dislocations (to be published)/20/. Therefore, if this $\alpha$ "component also disappears due to the addition of hydrogen, one could think that the interaction process of geometrical kinks on screw dislocations with hydrogen should also contribute of $\mathrm{SK}(\mathrm{H})$ relaxation as was suggested by vetter et al. (13) in order to explain SK2 component.

In this paper we have carried out experiments with high purity Iron doped with 
interaction of geometrical kinks on screw dislocations with hydrogen. Indeed, for

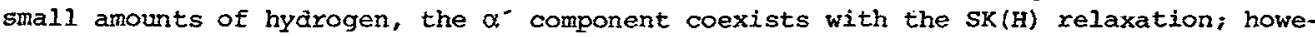
ver concerning large amounts of hydrogen, it seems clear that the interaction process of geometrical kinks with hydrogen contribute to the SK(H) relaxation.

\section{II.- EXPERIMENTAL PROCEDURE}

The measurements are made in an inverted pendulum described elsewhere $(8,14)$, on plates $(0.5 \times 5 \times 40 \mathrm{~mm})$ of CENG pure iron (15). We have applied an axial magnetic field of 200 oe in ordex to eliminate the effects of the magnetoelastic damping. The measurements were made at a mean strain amplitude of $5 \times 10^{-6}$.

The samples had been pre-deformed to 58 in traction at room temperature (RT) so as to suppress the $\beta_{\alpha}$ peak, and later they were deformed to $5 \%$ in torsion at RT.

Afterwards the hydrogen was introduced into the samples by electrolytic charging in a solution 58 of $\mathrm{H}_{2} \mathrm{SO}_{4}$ with a few drops of $\mathrm{CS}_{2}$ as catalytic agent, during 15 minutes at $400 \mathrm{~mA}$.

In order to obtain different concentrations of hydrogen, electrolytic charging was carried out by means of two procedures :

a) To obtain low concentrations, the charging was done at R.T., mounting the sample in the pendulum at RT as well and then cooling the cryostat.

b) To obtain high concentrations, the charging was done at $250 \mathrm{~K}$, mounting the sample in the pendulum at $78 \mathrm{~K}$, having cooled the cryostat previously.

\section{III.- RESULTS AND DISCUSSION.}

Firstly, we measured an internal friction spectrum of a pure iron sample which had been deformed to $5 \%$ by traction and $5 \%$ by torsion and we noticed only the $\alpha$ peak (fig.1a) wich is represented after eliminating the background. Then we charged the sample with a small amount of hyorogen and as a consequence of this, a relatively small SK(H) relaxation appeared in the internal friction spectrum (fig.1b). It can be observed that SK(H) relaxation corresponds almost exclusively to SK1 component ; furthermore, the $\alpha$ peak which was attributed to KPF on non-screw dislocations has disappeared almost completely except for the $\alpha^{-}$component at low temperatuxe, which was recently acknowledged as GKM on screw dislocations (to be published). Such $\alpha^{-}$peak does not decrease as can be observed in the levels of internal friction after eliminating the background. Both effects are also shown in the curves of the shear modulus (fig.2) where we can observe that after hydrogen charging (curve b), there is still a modulus defect below 20K, which corresponds to the $\alpha^{-}$component. On the other hand, the modulus defect associated with SK(H) relaxation is far smaller than the modulus defect associated with the former $\alpha$ peak (curve a). Such facts indicate that non-screw dislocations are hydrogen-blocked so as to prevent the $\alpha$ peak from appearing ; however the concentration of hydrogen atoms along the dislocation line is relatively low and thus $\mathrm{SK}(\mathrm{H})$ relaxation does not reach its maximum strength and is significantly shifted towards low temperatures, as it was predicted theoretically.

Afterwards we charged the sample with large amounts of hydrogen until we obtained an oversaturation above the equilibrium point $\left(\delta(\mathrm{SK})>40 \times 10^{-3}\right)$. Such oversaturation is the origin of some transitory effects on the internal friction spectrum which are not the subject of this paper and consequently will be dealt with in another paper. Due to annealing. at $240 \mathrm{~K}$ very slowly the system reaches an "equilibrium concentration", that is to say, transitory effects disappear and the basic outlook of the internal friction spectrum occurs recurrently and perfectly. Such internal friction spectrum is shown in fig.3a where a significantly developed SK(H) relaxation can be observed with an important SK2 component. However the $\alpha^{\circ}$ component of $\alpha$ peak has disappeared almost completely. Indeed, the curve of the corresponding shear modulus, $\mathrm{fig} .4 \mathrm{a}$, does not show any modulus defect at low temperatures; furthermore, if we compare curves $a$ and $c$ in fig. 4 we $c$ an notice that the modulus defect of SK(H) relaxation equals that of the former $\alpha$ peak. This indicates that under such conditions, $\mathrm{SK}(\mathrm{H})$ relaxation has reached its maximum equilibrium strength. Therefore, we could think after a first look at the results, that the disappearance of $\alpha$ component is related to the appearance of SK? component. However, such assumption is not totally correct, as we will now prove.

After a slow degassing at $240 \mathrm{~K}$ both peaks have decreased (fig.3b), but at the 
same time the $\alpha^{-}$component has developed at low temperatures. This effect can be clearly seen in the curve of the corresponding shear modulus, fig. $4 b$. If we analyse the curve of the modulus in detail and compare it with the curve obtained before degassing, it can be observed that the modulus defect corresponding to sk1 component has undergone almost no changes after and before the degassing process, whereas the modulus defect associated with SK2 component has decreased by an amount equal to the modulus defect associated with the $\alpha$ component. Indeed, the difference between curves $a$ and $b$ is approximately the same at $20 \mathrm{~K}$ and $130 \mathrm{~K}$ (indicated by means of arrows in fig.4).

These facts confirm the co-existence of $\alpha$ and the SK1 component shown in fig.1, and they seem to indicate that the appearanceand disappearance of the $\alpha^{\text {" component }}$ is not associated with such SK1 component. Therefore we could infer that the interaction process of hydrogen with the geometrical kinks on screw dislocations does not contribute to the SK1 component of the SK(H) relaxation.

Nevertheless, the appearance of the $\alpha^{-}$component is accompanied by the decrease of SK2 component by an equivalent amount, as it is shown by the modulus defect curves. Thus we could deduce that geometrical kinks on screw dislocations interact with hydrogen and produce a relaxation process that contributes to the SK2 component of the $\mathrm{SK}(\mathrm{H})$ relaxation. However, we must remark that such interaction is not the only process responsible for the origin of the SK2 component. In fact, if we compare the internal fxiction levels at low temperature we can see that intexnal friction in fig. $3 \mathrm{~b}$ is approximately the same as that shown in $\mathrm{fig} .1 \mathrm{~b}$, which indicates that the $\alpha^{-}$component has regained its maximum strength and therefore the geometrical kinks do not interact with hydrogen any more. However, SK2 component still has a noticeable strength that can only be attributed to the K.P.F. process controlled by the diffusion of pairs of hydrogen atoms (10). In this case, looking at the curves showing the modulus defect, we can estimate the contribution to the SK2 component as $40 \%$ by G.K.M. process and a 608 by K.P.F. process although such figures are not meaningful since they largely depend on the structure of the dislocation network and on the concentration of hydrogen clusters.

After obtaining these results, it seems to be clear that the presence of hydrogen atoms does not modify the mobility of geometrical kinks on screw dislocations. Two hypotheses can be proposed at this point.

a) The interaction of the geometrical kinks with hydrogen atoms is very weak. This could be due, among various factors, to a strong delocalization of the hydrogen atom in the dislocation environment caused by the stress field of dislocation core (16). Anyway, the stress field has the effect of perturbing the potential barrier between adjacent sites in a direction parallel to the dislocation line ard favours the pipe diffusion along dislocation lines (17).

b) The concentration of hydrogen atoms along the dislocation line is very small due to a low binding energy between screw dislocations and hydrogen atoms (18). On the other hand a high mobility of hydrogen atoms along the dislocation line could favour the formation of pairs of atoms clearing up of single atoms the dislocation line.

However, the experimental results show some intexaction between these pairs of hydrogen atoms and geometrical kinks on screw dislocations. In fact, diatomic clusters have a lower mobility and a binding energy with the dislocation higher than those of hydrogen atoms. Therefore, at low temperatures, hydrogen clusters behave as pinning points for the geometrical kinks on screw dislocations, making the $\alpha^{-}$component disappear. In the temperature range of the SK2 component of SK(H) relaxation, the diatomic clusters become mobile and at the same time capable of controlling the motion of the geometrical kinks. Such interaction process between geometrical kinks -hydrogen clusters is the cause of a relaxation process that we will name $\mathrm{SK}^{-}(\mathrm{H})$ and is superposed upon the SK2 component due to K.P.F. On non-screw dislocations, which is also controlled by the diffusion of clusters of hydrogen.

Such interaction of geonetrical kinks on screw dislocations and diatomic clusters of hydrogen can be extremely complex because of the various possible orientations of the pairs of atoms and the different types of geometrical kinks (19). Therefore this aspect should be studied more in detail in the future. However we can indicate now that the binding energy geometrical kink-hydrogen clusters $E_{B}(H-H)$ must be lower that the binding energy hydrogen cluster-non screw dislocation $\mathrm{E}_{\mathrm{B}}(\mathrm{H}-\mathrm{H})$, since the $\mathrm{SK}^{-}(\mathrm{H})$ component disappears through annealing at lower tempera- 
tures, and more quickly than SK2 component.

\section{IV. - CONCLUSIONS}

The co-existence of the $\alpha^{-}$component and the $\mathrm{SK}(H)$ relaxation that we observed allows us to come to the following conclusions :

a) The intexaction of geometrical kinks on screw dislocations and hydrogen atoms is null or very weak.

b) The SK1 component can be attributed totally to KPF on non-screw dislocations controlled by the diffusion of hyarogen atoms.

c) The interaction of geometrical kinks on screw dislocations and diatomic hydrogen clusters is noticeable and causes a relaxation peak that we will call $S K^{*}(H)$.

d) The SK2 component that we had noticed is the result of the superposition of two components :

$\mathrm{SK}^{-}(\mathrm{H})$ due to the process indicated above in $\mathrm{c}$.

SK2 itself, due to KPF on non-screw dislocations controlled by the diffusion of diatomic hydrogen clusters.

REFERENCES

1 .- Takita K., Sakamoto K.; Scripta Met. 10 (1976) 399

2 .- Moser P., Dufresne J.F., Ritchie J.G.; ICIFUAS-6, Ea. R. Hasiguti, University of Tokio Press (1977) 473

3 .- Ritchie I.G., Dufresne J.F., Moser P.; Phys. Stat. Sol. (a) 52 (1979) 331

4 .- Sakamoto K., Shimada M.; ICIFuAS-7, J. de Phys. 42 (1981) C5-109

5 .- Matsui H., Schultz H.; ICIFUAS-7, J. de Phys. 42 (1981) C5-115

6 .- San Juan J., Fantozzi G., Esnouf C., Vanoni F.; Proc. Hydrogen and Materials H-3, Ed. D. Azou, Paris 7-11 Juin (1982), 1B-8

7 .- San Juan J . Fantozzi G., Esnouf C., Vanoni F., Bernalte A.; ECIFUAS-4, J. de Phys. 44 (1983) c9-633

8 .- San Juan J.; Thesis INSA de Lyon (France) (1984)

9.- San Juan J., Fantozzi G., Nó M.L., Esnouf C., Vanoni F.; ICIFUAS-8, J. de Phys. 46 (1985) C1 $0-1 \quad 27$

10.- San Juan J ., Fantozzi G., Nó M.L., Esnouf C.; To be pubiished in J. of Phys.F Physics Metals.

11.- Hirth J.P .; Met. Trans. 11A (1980) 861

12.- Ritchie I.G.; Scripta Met. 16 (1982) 249

13.- Vetter K., Steeb H., Kronmūilex H.; Proc. Hydrogen in Metals H-2, Paris 6-10 Juin (1977) 2C-5

14.- Bouvier- Volaille J.J., San Juan J.; ECIFUAS-4, J. de Phys. 44, (1983) C9-353

15.- Vanoni F.; Thesis University of Grenoble (1973)

16.- Fukai Y., Sugimoto H.; Advances in Physics 34 (1985) 263

17.- Kiuchi K., Mc Lellan R.B.; Acta Met. 31 (1983) 1961

18.- Wert Ch. A.; Hydrogen in Metals II, Ed. by G. Alefeld and J. Völkl, SpringerVerlag, Berlin (1978) 305-330

19.- Seeger A., Wührich C.; II Nuovo Cimento 33B (1976) 38 


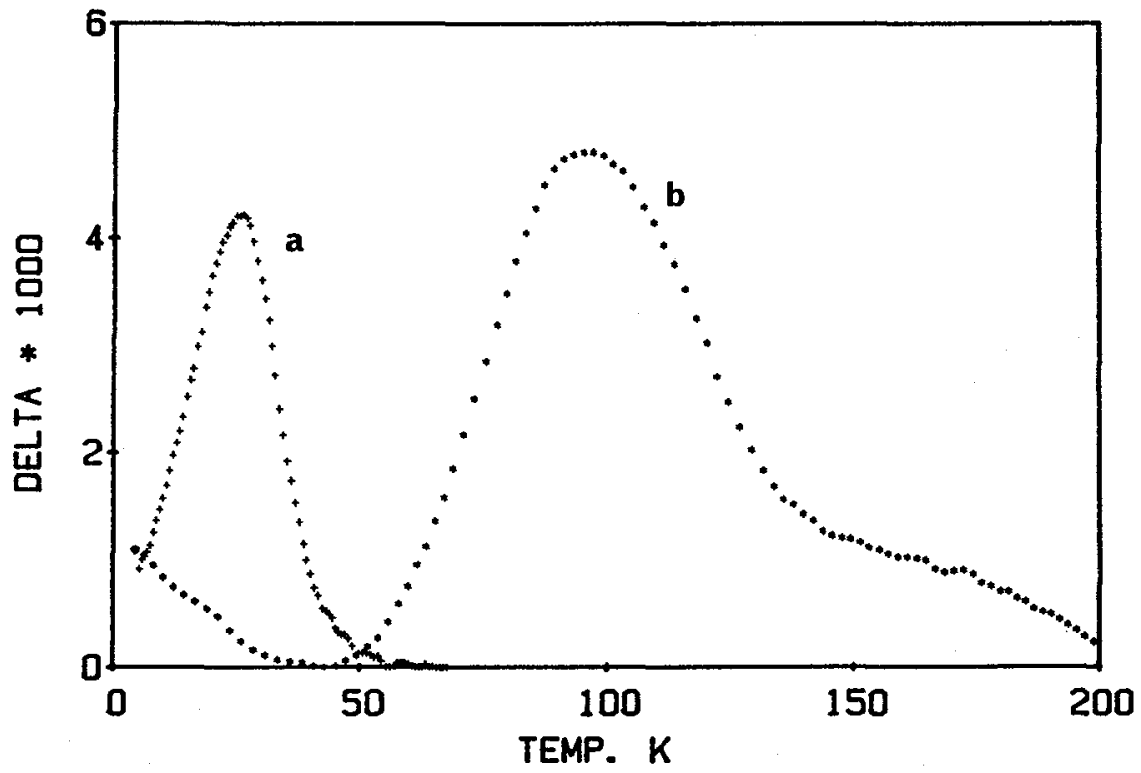

Fig.1.- Internal friction spectra before and after a weak hydrogen charging. The background has been eliminated for both spectra.

a) $\alpha$ peak before hydrogen charging

b) $\mathrm{S}-\mathrm{K}(\mathrm{H})$ peak in the same sample after a weak charging

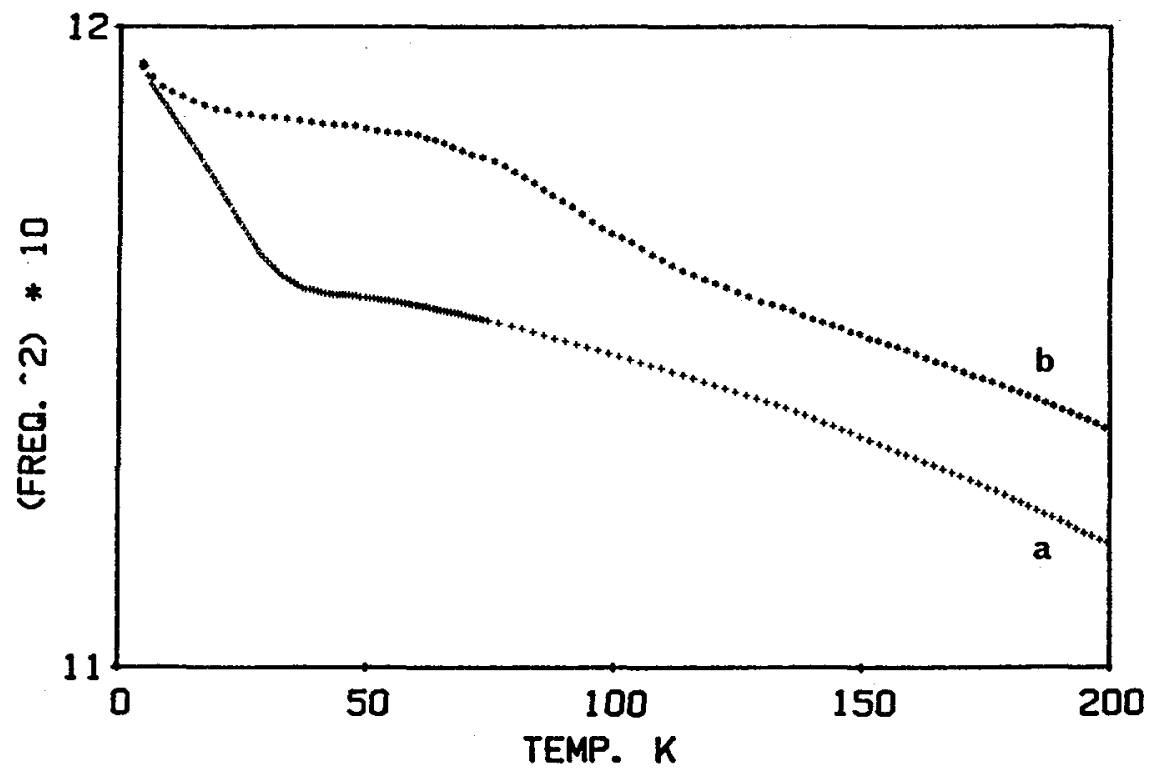

Fig.2.- Curves of the shear modulus corresponding to the spectra shown in the Fig.1. The curves are shifted for in such way their values at low temperatures coincide, making their comparison easier. 


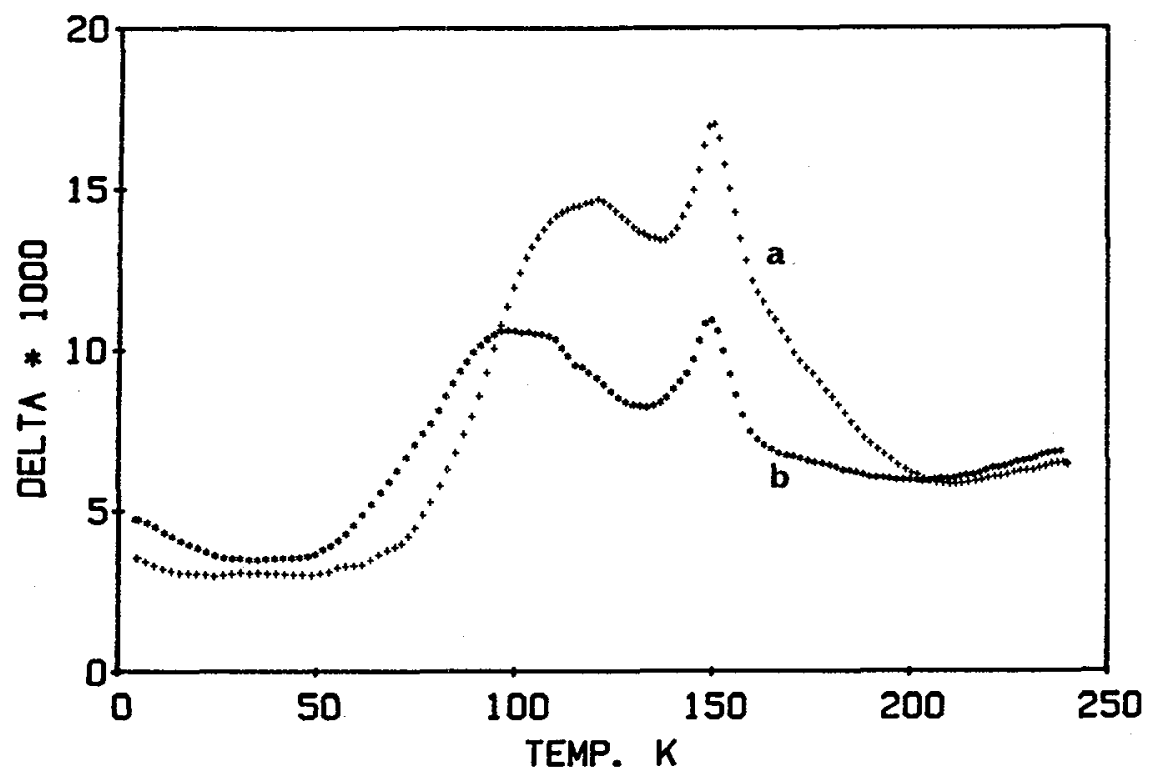

Fig.3.- Internal friction spectra after a stong hydrogen charging and annealing at $240 \mathrm{~K}$. a) S-K(H) relaxation after removal of the transitory effects by annealing at $240 \mathrm{~K}$. b) $\mathrm{S}-\mathrm{K}(\mathrm{H})$ relaxation after a long annealing at $240 \mathrm{~K}$.

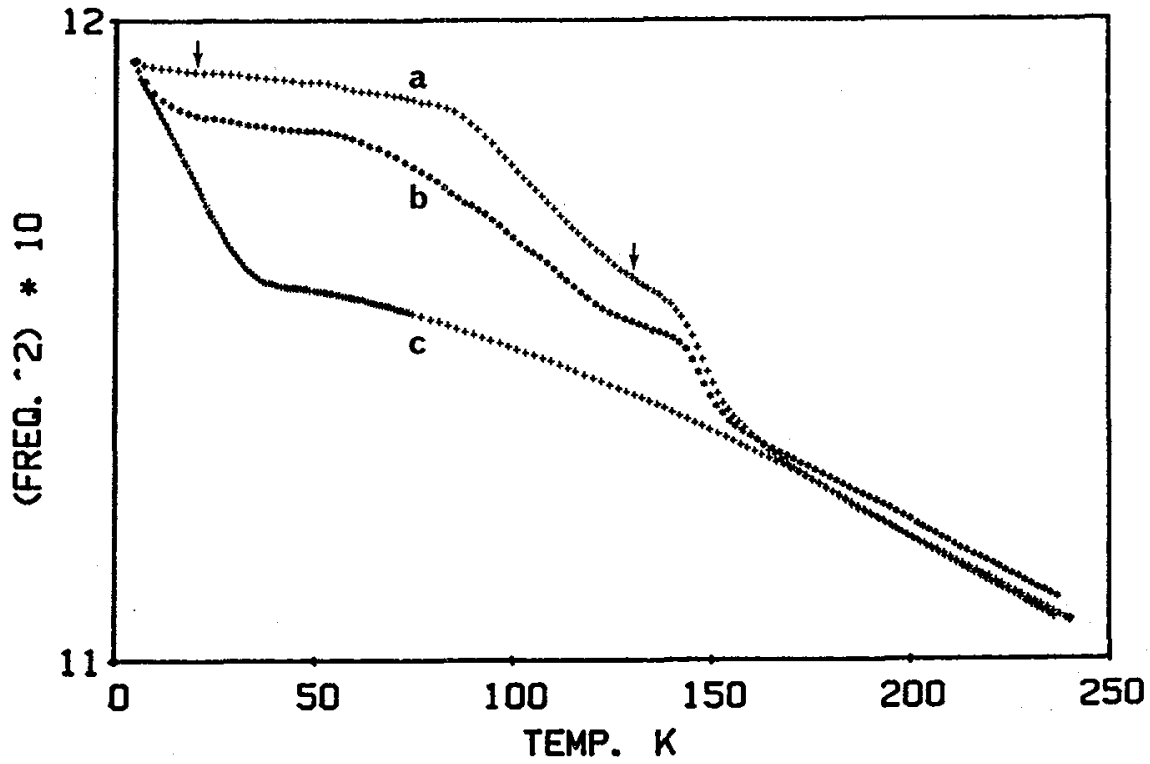

Fig.4.- Curves of the shear madulus corresponding to the spectra shown in Fig. 3 . These curves have not been normalized. They are just shifted for in this way their value at low temperatures coincide, making their comparison easier.
a) After strong hydrogen charging
b) After a long annealing, at $240 \mathrm{~K}$
c) Curve of shear modulus of the former $\alpha$ peak 